\title{
Creation of a Table of Primes in Successive order
}

\author{
Nikolaos Vrettos
}

\begin{abstract}
The sequence of prime numbers has been one of the problems that have occupied the minds of mathematicians for the last centuries. Perhaps there is no formula that gives this sequence, number by number, or it is very hard to find, but this sequence could arise from one or more sequences of composite numbers and the gaps they leave in the set of integers. This work aims at something quite simple: To categorize all odd composite numbers, smaller than a given number $N$ and greater than number 1 , with the press of a button, into three types of arithmetic progressions that is, to make possible the creation of an algorithm and thus, obtain the results. That is, to have directly all odd composite numbers which are smaller than the given number N. So having the set of odd numbers and having created a subset of the composite ones, it is easy then to give to the computer, the command to delete all the existing members of composites from the set of the odd numbers as they have been categorized in the subset of the found composites and make their set difference. This set difference can have as members only prime numbers, because the set of the odd numbers greater than number 1, consists only of primes and composites.
\end{abstract}

\section{I.The Conditions And The Creation Of The Algorithm}

The only condition required, is to have all the prime numbers $p 1, p 2, p 3, \ldots p s$ starting from number 3 up to the square root of the number $N$ that has been given (prime $p s$ ), and the primes included between $p s$ and the quotient of the division of $\mathrm{N}$ by $p s$, ( $\mathrm{N} / p s)$. For example, for number 16,128, it is essential to know only the primes from 3 to 113 and furthermore the primes 127, 131, 137 and 139.

The steps for the creation of an algorithm giving the table of primes up to $N$ can be outlined as follows. (The proof of necessity for making these steps is described in the next paragraphs).

1. Select number 2 and all the odd numbers from 3 and up to $\mathrm{N}$ in one set.

2. Select all primes up to the $\operatorname{sqrt}(\mathrm{N})$ and the following the last prime (ps) selected, and up to (N/ps).

3. Make 3 categories of arithmetic progressions under the condition, for each one its last term to be equal to, or smaller than $\mathrm{N}$.

3a. One set of arithmetic progression with terms all the odd multiples of 3 , that is $9,15,21$, .. etc, having a constant difference 6 .

3b. Sets of arithmetic progressions for each prime $p$, up to the $\operatorname{sqrt}(\mathrm{N})$, with first term the square of the prime selected and difference $6 p$ e.g.

$\mathrm{p} 1 \wedge 2, \mathrm{p} 1 *(\mathrm{p} 1+6), \mathrm{p} 1 *(\mathrm{p} 1+12), \mathrm{p} 1 *(\mathrm{p} 1+18), \ldots$

$\mathrm{p} 2 \wedge 2, \mathrm{p} 2 *(\mathrm{p} 2+6), \mathrm{p} 2 *(\mathrm{p} 2+12), \ldots$ and so on

The multiple appearance of common terms among them will be arranged in step 4.

3c. Sets of arithmetic progressions of the product $p 1 p 2$ of each prime number and its consecutive prime, starting from 5.7=35, up to the product of $p s$ with the last in the series prime greater than $p s$ and with difference $6 p$. Omit the consecutive prime and skip to the next consecutive prime, if the initial difference between $p 2$ and $p 1$ is divisible by 6 .

$\mathrm{p} 1 * \mathrm{p} 2, \mathrm{p} 1 *(\mathrm{p} 2+6), \mathrm{p} 1 *(\mathrm{p} 2+12), \mathrm{p} 1 *(\mathrm{p} 2+18), \ldots$

$\mathrm{p} 2 * \mathrm{p} 3, \mathrm{p} 2 *(\mathrm{p} 3+6), \mathrm{p} 2 *(\mathrm{p} 3+12), \ldots$ and so on.

The multiple appearance of common terms among them will be arranged in step 4

4. Gather in one union of sets all the terms of the arithmetic progressions under $\mathbf{3 a}, \mathbf{3 b}$ and $\mathbf{3} \mathbf{c}$ in one set.

5. Find the set difference between the set of step 1 and the set of step 4.

This Set Difference' S Members, Are All The Primes, One By One, Up To Number N

All this seems quite naif. But is it effective? What is useful in it?

It has nothing to do with Eratosthenes' sieve as it does not examine one number to find its divisors but it searches for all the primes up to that number.

It has a mathematical structure which can lead to an algorithm.

The calculations to find the primes do not lead to huge numbers in relation with $\mathrm{N}$ as it happens with the existing primality tests.

It gives the exact number of primes up to a number $\mathrm{N}$ and not a statistic measure of it as it happens now with $\mathrm{N} / \log \mathrm{N}$.

As a consequence of this, the algorithm applied on $(\mathrm{N}+2)$ is by itself, a secure primality test for any odd number next to $\mathrm{N}$. 
It does not skip primes as it happens with Mersenne' s primes.

It can go quickly to complete tables of very large primes, as the data of the known primes can lead to numbers as great as their square. For example with known primes up to 97 , we can go to primes up to 9,409 - right after to primes up to $88,529,281$ and right after to primes up to the square of $88,529,281$ and so on.

\section{II.Categorization Of Composites}

We distinguish three types of odd numbers:

-Numbers divisible by three (3) of the form $6 n+3 \quad(n=0,1,2,3,4 \ldots$ etc)

-Numbers with remainder five (5), after their division by six (6). Form $6 n+5 \quad(n=0,1,2,3,4 \ldots$ etc)

-Numbers with remainder seven (7), after their division by six (6). Form $6 n+7 \quad(n=0,1,2,3,4 \ldots$ etc)

Proof that this categorization includes all odd numbers from 3 to $\mathrm{N}$

For the same value of $n$, the number of the form $(6 n+3)$ deducted from $(6 n+5)$, gives two (2), the least distance between two odd numbers.

The same holds for $(6 n+5)$ deducted from $(6 n+7)$. This leads to a constant sequence of odd numbers increasing by 2 .Now if on $(6 n+7)$, the number 2 is added, we get $6 n+9$ and so go back to the form $(6 n+3)$, for the next $n$ in sequence. If another 2 is added, the number becomes $6 n+11$ which is $(6 n+6+5)$ and so go back to the form $(6 n+5)$ for the next $n$ in sequence. If another 2 is added, we get $6 n+13$ which is $(6 n+6+7)$ and so go back to the form $(6 n+7)$ for the next $n$ in sequence and so on. This proves that the present categorization includes all the requested odd numbers, greater than 1: Primes and composites.

Proof of the categorization of the product of an odd composite with two factors

Therefore, all the odd numbers non divisible by 3 , including composites as well, must be either of the form $6 n+5$, or of the form $6 n+7$ and the odd composites non divisible by 3 , can only be the result of multiplying two or more numbers of these forms. So, if the first of the two factors of a composite is a prime number, as it has been defined here, there are only three possibilities:

1) To multiply a prime of the form $(6 n+5)$ with a number of the form $(6 n+7)$.

This gives a sum of two numbers, the first one divisible by 6 plus 35 , equal to $6.5+5$

So the resulting composite number is always of the type $(6 n+5)$.

2) To multiply a prime of the form $(6 n+5)$ with also a number of the form $(6 n+5)$.

This gives a sum of two numbers, the first one divisible by 6 and the second 25, equal to $6.3+7$

So the resulting composite number is always of the type $(6 n+7)$.

3) To multiply a prime of the form $\{6 n+7)$ with also a number of the form $(6 n+7)$

This gives a sum of two numbers, the first one divisible by 6 and the second 49 , equal to $6.7+7$

So the resulting composite number is always of the type $(6 n+7)$.

Regarding the above, it becomes clear, that multiples of these forms differing by 6 , or by multiples of 6 , belonging to the same form $(6 n+5$ or $6 n+7)$, are always of the form $(6 n+7)$. For example $31.37=6.190+7$, (because $37-31=6$ ), $29.41=6.197+7$, (because $41-29=12$ and regardless of the fact that $29=6.4+5$ and $41=6.6+5$ ), while $31.35=6.180+5$ and $29.31=6.149+5$ etc

\section{III.The sequence of composites}

For the form $(6 n+7)$ we define each first term of each progression of composite numbers, to arise from only two factors, with the first factor to be a prime number and the second factor the same prime as well, (that is the square of the prime), increasing by 6 for every second factor of every next term of the progression. For the form $(6 n+5)$ and for the first term of the progression of composites, the same prime is selected as the first factor and the next to come from the series of primes, as the second factor increasing by 6 for every next term and up to the square root of the given number, excluding only second factors differing from the first ones by multiples of 6 . That's because, the products of them have already been written with the composites of the form $(6 n+7)$.

For example, for number 979 apart from 2 and 3, we get the primes 5, 7, 11,13,17, 19, 23, 29 and 31. The quotient 979:31=31,580645 so we have no additional primes. In order to find the composites of the forms $(6 n+7)$ and $(6 n+5)$, we get the following initial terms of the progressions for each form with the second factor of each initial term to be increasing by 6 :

$5.5,7.7,11.11,13.13,17.17,19.19,23.23,29.29,31.31$ for the form $(6 n+7)$

$5.7,7.11,11.13,13.17,17.19,19.23,23.31,29.31$ for the form $(6 n+5)$

Number 31 as the next prime after 29 , is selected for 23.31, instead of 23.29 because $29-23=6$ and consequently 23.29 is the second term after 23.23 , number which has already been recorded with the progression with initial term 23.23 belonging to the form $(6 n+7)$. So: The sequence of the odd composites divisible by three (3) is a progression starting always from $3.3=9$ and with a constant difference the number 6 , that is $9,15,21,27 \ldots$ etc The sequence of the odd composites of the form $(6 n+5)$ is a progression, with terms the product of two consecutive primes, with a difference between them not divisible by 6 and always starting from $5.7=35$. In arithmetic terms it might be 5.7, 5.13, 5.19, 5.25, 5.31 ... with a difference of $6.5=30$ from each term 
to its next, or $7.11,7.17,7.23,7.29,7.35,7.41 \ldots$ etc. with a difference of $6.7=42$ from each term to the next one of the progression.

The sequence of the odd composites of the form $(6 n+7)$ is a progression, with a starting term the square of the primes up to the square root of the given number and starting always from $5.5=25$. In arithmetic terms it might be for example for the prime $5,5.5,5.11,5.17,5.23 \ldots$ with a difference of $6.5=30$ from each term to its next one of the progression.

Proof that this categorization includes all the composites up to N: No composite smaller than N, with two factors, with the second factor equal or greater than the first, can have its first factor (always a prime according to our categorization) greater than $\operatorname{sqrt}(\mathrm{N})$. So every composite of two factors is equal to, or smaller than $\mathrm{N}$, with its first factor a prime number and with its second factor (prime or composite) always greater than its first and with a constant flow of numbers (forms $6 n+3,6 n+5$ and $6 n+7$ ) as above.

The increase of the second number of every term by 6 each time, secures that it remains in its form $(6 n+5)$ or $(6 n+7)$ and that the difference between two composites of the same form having as a common divisor the same prime as it has been defined, is the least one or, in other words, between these two terms it is impossible that a composite of the same form with a divisor the selected prime, could be inserted. If any other integer apart from 6 was added to the second number, it would result to the change of the form of the term, that is, the next term to become an even number, the next term to become a number divisible by 3 , the next term to become one composite of the form $(6 n+7)$ when a term of the same progression of the form $(6 n+5)$ has preceded and vice - versa, or simply to remain in the same form with the other terms of the progression, if the added integer is a multiple of 6 , but having skipped some terms (that is composites of the same form) of the progression. This leads to the conclusion that this categorization of composites always leads to a composite of the same form, that the integers inserted between two successive terms of one of the three forms, must either belong to one of the two other forms or be an even number or a prime and that the way of the formation of the composite numbers in progressions, is absolutely fixed.

\section{IV.Conclusion}

Having all this in mind and the facilities offered by the digital technology today, given a number and in order to find a full list of primes up to this number, it is enough to give the computer the command to store in a set all the odd numbers from the number 3 to the given number, calculate and store a second set of results of the union of arithmetic progressions as outlined above and finally find the set difference of the two sets, which is a set of all terms of prime numbers up to the given number except number 2 .

Primes up to 1000

\section{V.Examples}

The numbers greater than 1 and up to 1000 are 999

The odd numbers from 3 to 1000 are 499

The even composite numbers up to 1000 are 499

The square root of 1000 is 31,622776

We have the primes $2,3,5,7,11,13,17,19,23,29$ and 31 up to the square root of 1000 . No additional primes are taken under consideration as $1000 / 31=32,258064$

The odd composite numbers divisible by 3 (form $6 n+3)$, from $3.3=9$ to $3.333=999$ are 166

The progressions of the form of numbers $6 n+7$ are as follows with first term:

5.5=25: $\quad$ (progression difference 6.5 $=30$ )

$25,55,85,115,145,175,205,235,265,295,325,355,385,415,445,475,505,535,565,595,625,655,685$, $715,745,775,805,835,865,895,925,955,985$

7.7=49: $\quad$ (progression difference $6.7=42$ )

$49,91,133,175,217,259,301,343,385,427,469,511,553,595,637,679,721,763,805,847,889,931,973$

11.11=121: (progression difference 6.11=66)

$121,187,253,319,385,451,517,583,649,715,781,847,913,979$

13.13=169: (progression difference $6.13=78$ )

169, 247, 325, 403, 481, 559, 637, 715, 793, 871, 949

17.17=289: (progression difference $6.17=102$ )

289, 391, 493, 595, 697, 799, 901

19.19=361: (progression difference $6.19=114$ )

361, 475, 589, 703, 817, 931

23.23=529: (progression difference 6.23=138)

$529,667,805,943$

$29.29=841:($ progression difference $6.29=174)$

841 
31.31=961: (progression difference 6.31=186)

961

The total number of the terms of the above arithmetic progressions is 100 . The terms with multiple appearance in this case they are 14, so we have 100-14=86 composites and the union of the sets corresponding to the arithmetic progressions of the form $(6 n+7)$, has 86 members.

The progressions of the form of numbers $6 n+5$ are as follows with first term:

5.7=35: $\quad$ (progression difference 6.5=30)

$35,65,95,125,155,185,215,245,275,305,335,365,395,425,455,485,515,545,575,605,635,665,695$,

$725,755,785,815,845,875,905,935,965,995$

7.11=77: (progression difference $6.7=42$ )

77, 119, 161, 203, 245, 287, 329, 371, 413, 455, 497, 539, 581, 623, 665, 707, 749, 791, 833, 875, 917, 959

11.13=143: (progression difference $6.11=66$ )

143, 209, 275, 341, 407, 473, 539, 605, 671, 737, 803, 869, 935

13.17=221: (progression difference $6.13=78$ )

$221,299,377,455,533,611,689,767,845,923$

17.19=323: (progression difference 6.17=102)

$323,425,527,629,731,833,935$

19.23=437: (progression difference $6.19=114$ )

$437,551,665,779,893$

23.31=713: (progression difference 6.23=138)

$713,851,989$

29.31=899: (progression difference 6.29=174)

899

The total number of this form $(6 n+5)$, of the above arithmetic progressions is 94 , The terms with multiple appearance in this case they are 14 so we have $94-14=80$ composites and the union of the set corresponding to these arithmetic progressions has 80 members.

Now we are ready to count primes and composites up to 1000 that is 999 numbers because number 1 is neither a prime, nor a composite.

Composites divisible by 2 are 499

Composites divisible by 3 are 166 (odd ones)

Composites of the form $(6 n+5)$ are 80

Composites of the form $(6 n+7)$ are 86

This makes a sum of 831 composites which deducted from 999 gives 168 primes which is correct.

Primes up to 101

The numbers greater than 1 and up to 101 are 100

The odd numbers from 3 to 101 are 50

The even composite numbers up to 101 are 49

The square root of 101 is 10,049875

We have the primes 2,3,5,7 up to sqrt(101) and the primes 11 and 13 because 101:7=14,428571. However the prime 13 is not taken under consideration because $13-7=6$ and so the term $7.13=91$ has been already a term of the progression with initial term $7.7=49$ and constant difference $6.7=42$

The odd composite numbers divisible by 3 (form $6 n+3$ ) from $3.3=9$ to $3.33=99$ are 16

The progressions of the form of numbers $(6 n+7)$ are as follows:

$5.5=25$ (progression difference $6.5=30$ )

$25,55,85$

$7.7=49$ (progression difference $6.7=42$ )

49,91

That makes a total of 5 composites of the form $(6 n+7)$ with no common terms

The progression of the form of numbers $(6 n+5)$ are as follows:

$5.7=35$ (progression difference $6.5=30$ )

$35,65,95$

$7.11=77$ (progression difference $6.7=42$ )

$77(7.17=119$ is greater than 101$)$

And that makes a total of 4 composites of the form $(6 n+5)$ with no common terms

So the primes up to 101 among 100 primes and composites from 2 to 101 are:

100 numbers minus 49 (even composites) $=51$ numbers

51 numbers minus 16 numbers divisible by 3 , makes 35 primes and composites of the forms $(6 n+7)$ and $(6 n+5)$ $35-5-4=26$ primes up to 101 which is correct.

Or, in an even more simple way of appearance and without the need of using primes greater than $p s$ up to N/ps, 
(here, 11 after 7 ) by the use of the following progressions:

$3.3,3.5,3.7,3.9,3.11,3.13, \ldots \ldots .3 .31,3.33$ (16 terms with progression difference 6$)$

$5.3,5.5,5.7,5.9,5.11,5.13,5.15,5.17,5.19$ (9 terms with progression difference 10$)$

$7.3,7.5,7.7,7.9,7.11,7.13$ (6 terms with progression difference 14$)$

Among them, there are 6 common terms, so their union has $16+9+6=31$, minus 6 , makes a total of 25 odd composites, which deducted from a total of 51 numbers gives us again 26 primes. In fact, the above progressions according to our categorization are:

3. $(6.0+3), 3 .(6.0+5), 3 .(6.0+7), 3 .(6.1+3), 3 .(6.1+5), 3 .(6.1+7) \quad \ldots \ldots \ldots . . .3 .(6.4+7), 3 .(6.5+3)$

$5 .(6.0+3), 5 .(6.0+5), 5 .(6.0+7), 5 .(6.1+3), 5 .(6.1+5), 5 .(6.1+7), 5 .(6.2+3), 5 .(6.2+5), 5 .(6.2+7)$

7.(6.0+3), 7.(6.0+5), 7.(6.0+7), 7.(6.1+3), 7.(6.1+5), 7.(6.1+7)

(This article is an extract of a never published work of mine of 2009 : "classification of composite numbers. An approach to Primes").

\section{Author: Nikolaos Vrettos}

Thessaloniki Greece

Article's Mathematics Subject Classification 2010 : 11-XX 11 N05 